\title{
TOWED GEO-ELECTRODE ARRAYS FOR ANALYSIS OF SURFACE WATER GROUNDWATER INTERACTION
}

\author{
David A. Allen - National Centre For Groundwater Management - Univ. of Tech., Sydney, Australia \\ Noel P. Merrick - National Centre For Groundwater Management - Univ. of Tech., Sydney, Australia
}

\begin{abstract}
High productivity analysis of surface water groundwater interaction is possible using GPS positioned vertical electrical conductivity imaging along with depth recording. Short submerged geoelectric arrays provide a great deal of detail right at the base of surface water bodies; however they cannot be used in treacherous waterways with debris scattered through them. Long floating arrays can be towed through such waterways and have the additional benefit of greater depth of exploration. Floating arrays also can have reasonable resolution at the base of surface water bodies provided that they are designed optimally and that data from them is inverted effectively. Array design must optimize a balance of signal strength maximization, exploration depth resolution, weight and drag minimization, crosstalk and current leakage minimization and simple, minimal response to three dimensional heterogeneity. Pragmatic interpretation of the huge volumes of data that are typically acquired is best achieved using three dimensional 'ribbon' images. Prominent features of interest are, in most cases related to groundwater salinity. Low conductivity anomalies often indicate fresh surface water seepage into more saline groundwater. High conductivity anomalies that intersect the bases of surface water bodies usually indicate saline inflow to the surface water bodies.
\end{abstract}

\section{Introduction}

Geo-electric arrays have been used for many years for electrical conductivity imaging on land; however it is only with the advent of GPS positioning and high volume memory storage that continuous geo-electric imaging on water has become popular. Such imaging has been typically conducted using many of the same parameters and innovations as used for ground based surveys. This paper aims to demonstrate new parameters and innovations suited to waterborne imaging.

\section{Geo-electric Array Design}

A new configuration of geo-electric array is defined here - the Allen Exponential Bipole Array. It has two linear (or point) transmitter electrodes. On one side of those electrodes, a set of exponentially spaced receiver electrodes is laid out. Consecutive receiver electrodes are used in pairs along with the transmitter electrodes in order to measure electrical conductivity at approximately exponentially spaced depths. A balance of several parameters can be optimized when designing arrays for surface water groundwater interaction investigation. 


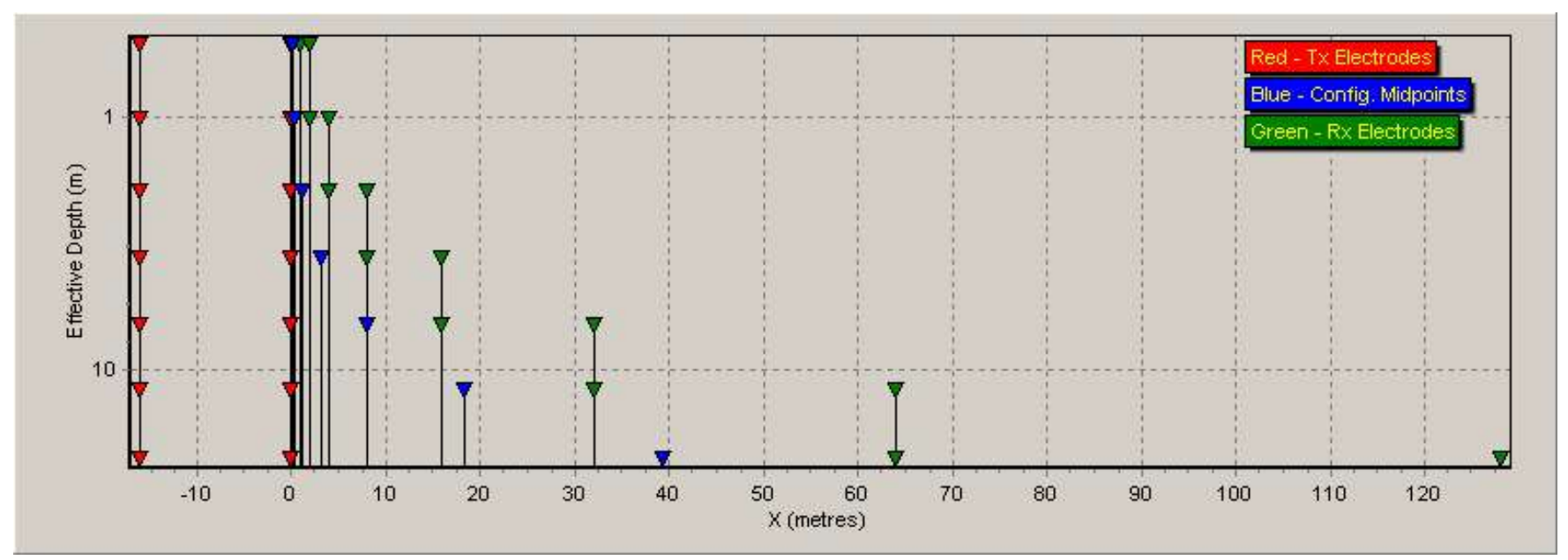

Figure 1.: $144 \mathrm{~m}$ Allen Exponential Bipole array electrode configurations plotted at their respective effective depths.

Discussion of the new array configuration and the most important of those parameters follows.

\section{Depth Resolution}

The nature of potential field geophysics results in an inverse exponential change of resolution with respect to depth. In order to make best use of available resolution for a given noise level, geoelectric arrays need to sample depth in approximately exponential increments. Traditional arrays such as the Schlumberger and Wenner arrays do this as do Allen Exponential Bipole Arrays (see Figure 1). In contrast, dipole dipole arrays sample depth in an almost linear manner. This contrast is evident from comparison of Figures 2 and 3. Usually, most depth resolution is required just under the base of surface water bodies rather than at the surface of them. Submersible geo-electric arrays facilitate this type of depth resolution distribution.

\section{Signal Strength}

Because towed arrays transmit from two electrodes while receiving simultaneously from many electrode pairs, pairs with poor signal strength cannot be stacked any longer than pairs with high signal strength. An optimal array for towing would therefore deliver good signal and minimal decay of signal strength with respect to sampled depth. A graph of signal strength versus effective depth for various arrays is presented in Figure 4. Wenner and some Schlumberger arrays produce the strongest signal while the Dipole Dipole array produces the weakest and fastest decaying signal strength of all commonly used arrays. Should linear transmitter electrodes be used in an Allen Exponential Bipole array, then contact resistance can be reduced, greatly increasing signal strength particularly at more distant electrodes. Efficient processing of data collected from such linear electrodes is possible - the author plans to publish the required mathematics soon. 


\section{Floating 5m Dipole Array (TxSpacing 5m)}

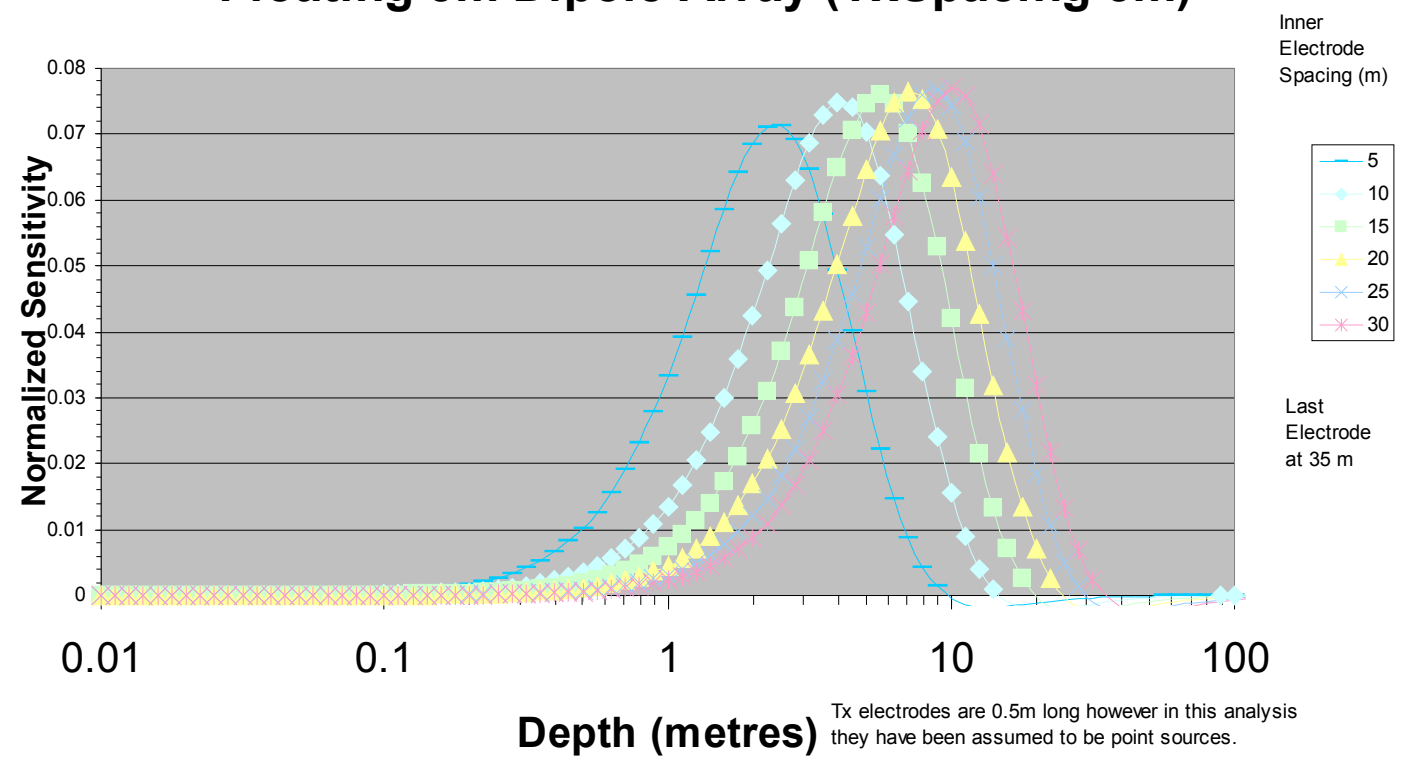

Figure 2.: Distribution of signal with respect to depth for a Dipole Dipole Array. Note that a small range of depths is sampled very well. In practice, the deeper sampling configurations rarely receive enough signal to be of much use.

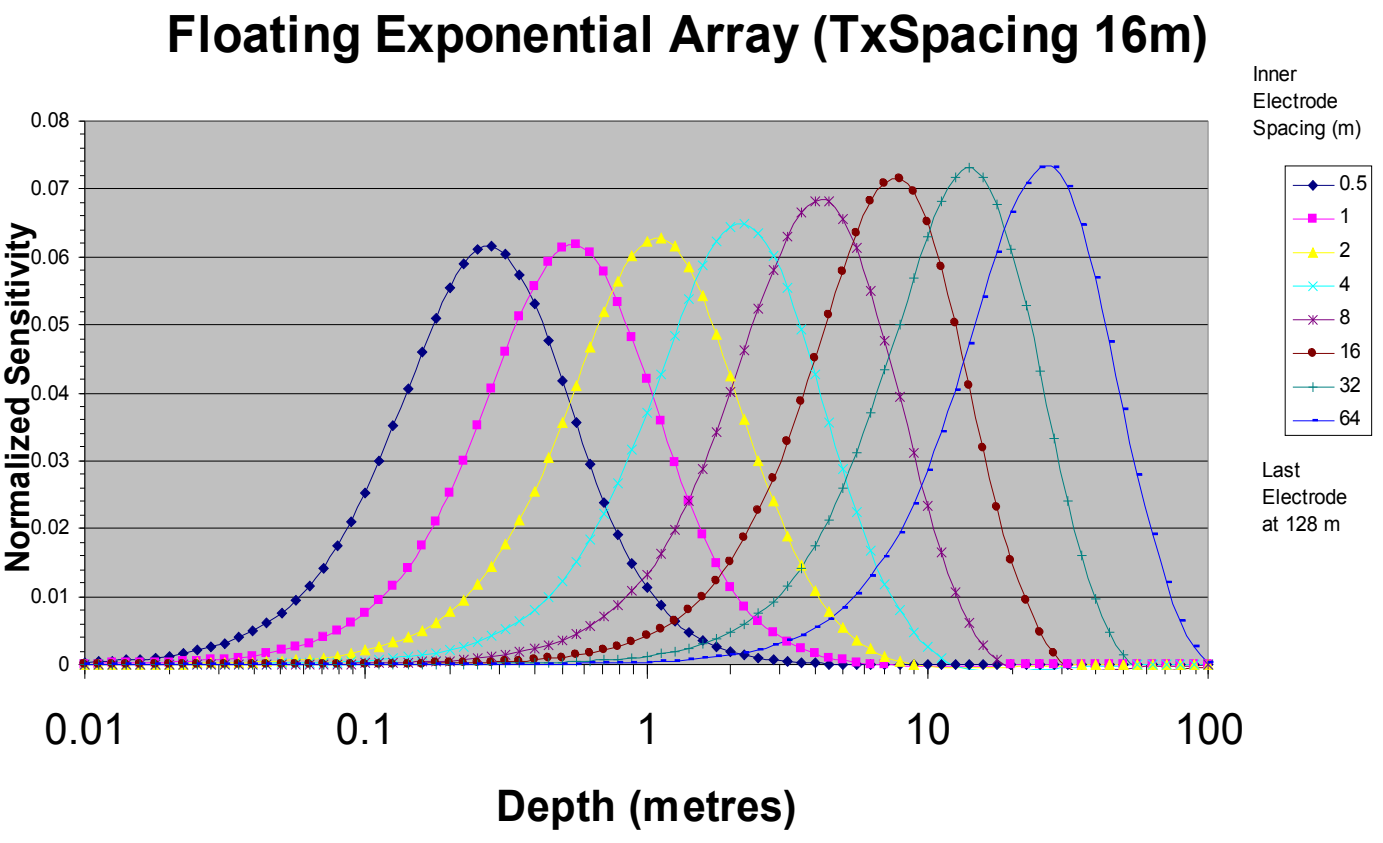

Figure 3.: Distribution of signal with respect to depth for an Allen Exponential Bipole Array. In contrast to the Dipole Dipole array, distribution of sampled depths is very even. 


\section{Signal Strength vs Effective Depth}

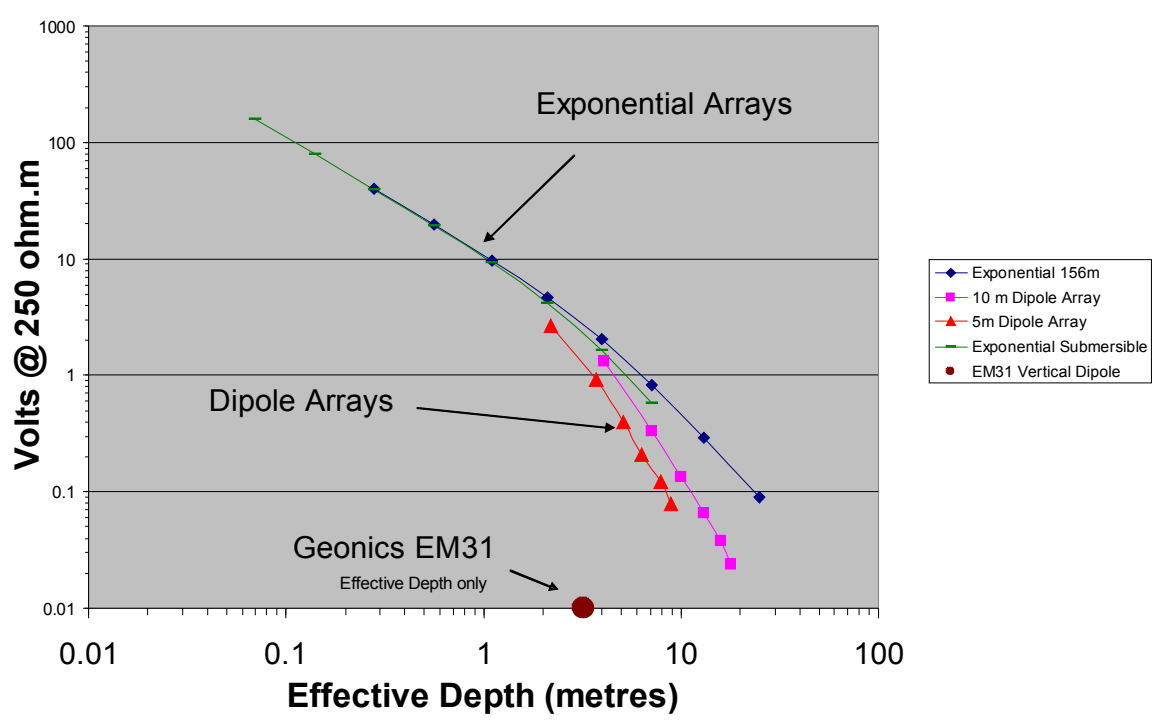

Figure 4.: Signal strength versus effective depth for various arrays over a halfspace with resistivity of $250 \mathrm{ohm} . \mathrm{m}$. The $156 \mathrm{~m}$ exponential array has a transmitter electrode separation of 16 metres and receiver electrodes spaced at $2^{\wedge} n$ where $n$ ranges from -1 to 7 . The exponential submersible array has a transmitter electrode separation of 8 metres and receiver electrodes spaced at $2^{\wedge} n$ where $n$ ranges from -3 to 5 . Geonics EM31 vertical dipole effective depth has been included simply for convenient comparison.

\section{Weight, drag, crosstalk and current leakage}

The reason that the Allen Exponential Bipole Array has been utilized in preference to Wenner and Inverse Schlumberger configurations, which have higher signal strengths, is that the heavy transmitter electrodes can be placed close to the towing device and that the number of wires needed in the cable decreases rapidly with distance from the second transmitter electrode. This means that the cross section area of the cable (and float if installed) may quickly diminish as it passes away from the towing device. A much more manageable cable weight results. Streamlining of the cable also becomes possible as a result. This reduces cable drag which in turn makes the cable more navigable because a cable with high drag, particularly at a great distance from the towing device, will tend to slew sideways rather than follow its course when towed along meandering watercourses. Crosstalk and current leakage problems are very difficult to manage in cables designed for use in water. In the Allen Exponential Bipole array, the lengths of high voltage wires are minimized resulting in less potential for current leakage and crosstalk problems.

\section{Simple minimal response to three dimensional heterogeneity}

The effect of three dimensional heterogeneity on various arrays can be studied using signal contribution element images. Such images show that most signal results from closely spaced transmitter - receiver electrode pairs. Therefore the ideal array for simplifying and minimizing the effect of three dimensional heterogeneity is the theoretical Pole - Pole array configuration which has two electrodes at infinity. The Allen Exponential Bipole Array has only one closely spaced pair in each of the shallower configurations - they approximate the Pole - Pole configuration. Midpoint of response of each configuration can also be calculated using signal contribution element analysis - see Figure 1. 


\section{Field Logistics}

Towed array surveys are requested at sites where canal seepage is problematic, where transmission losses from rivers need to be studied, and where saline inflow, acid inflow (from acid sulfate soils) or other pollution inflow into rivers or drains is occurring. These sites rarely offer ideal navigation and innovative array towing solutions usually need to be implemented. Equipment used, including the geo-electric arrays, typically needs to be light, rugged and streamlined. Figure 5 presents some of the solutions implemented so far. Field logistics, rather than geophysical limitations, is usually the factor that determines the viability of towed geo-electric array surveys.

A competing technology - Towed Zonge NanoTEM also can be used for surveying under large water bodies. It requires towing of a large (approx $8 \times 8 \mathrm{~m}$ ) loop. NanoTEM systems cannot resolve detail right at riverbeds as well as appropriately designed geo-electric arrays due to ambiguities in response from shallow features.

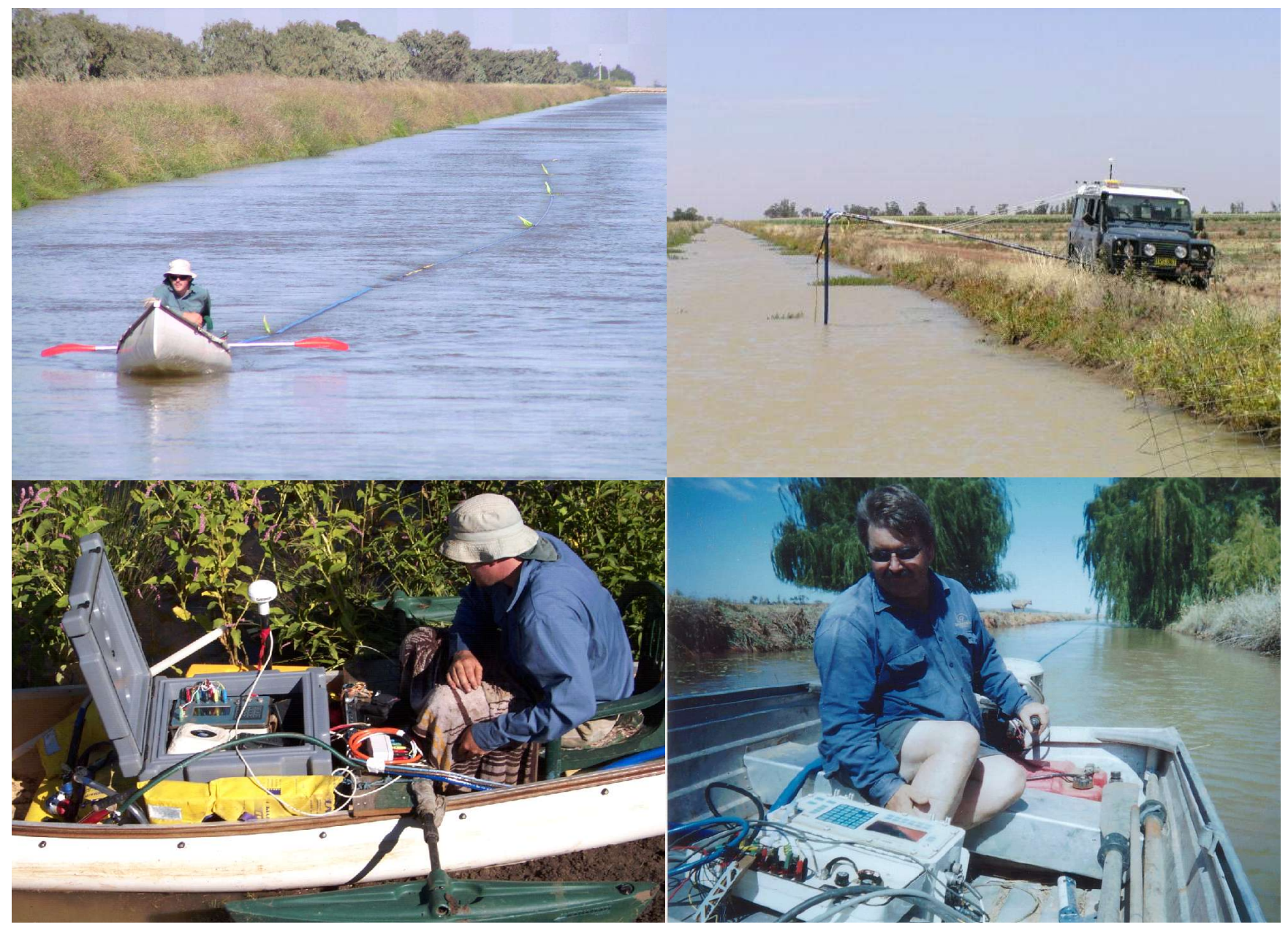

Figure 5.: Equipment for towed geo-electric surveying from surface water bodies. Small watercraft such as canoes with outboard motors are appropriate in canals which typically contain numerous obstacles over which the craft must be lifted either by hand or with a small crane. In canals with lots of weed, which quickly stops outboard motors, a boom extending from a $4 \mathrm{wd}$ is most appropriate for towing arrays. Electronics suitable for operating the arrays is produced by ABEM - Terraohm RIP924, Iris Instruments - Syscal Pro (lower left), Zonge - GDP32 (lower right) and AGI - Supersting R8 Marine. 


\section{Presentation}

Tens of megabytes of data can easily be collected by GPS tracked towed geo-electric arrays in a day. Presentation of that data is only feasible once it is in a form in which it can be efficiently georeferenced by the viewer. Because of this, 3D ribbon imaging has been developed. Examples are

presented in Figures 6,7 and 8. Interactive viewing in an OpenGL interface further enhances usability of the data.

Any data can be imaged using half space apparent resistivity equations; however floating array data is best inverted using horizontal layer inversion. Two dimensional inversion such as displayed in Figure 7 is excessively time consuming and ill posed to towed array data. The horizontal ripple evident in Figure 8 usually results. Initial models fed to inversion should be adjusted using the measured water depths. Inversion routines should not however be forced to honor the water depth especially where surface water stratification is anticipated or where water depth is laterally variable (i.e. most rivers and canals). Forcing inversion to honor the measured water depth in those situations would produce neater more impressive images but they would have artifacts in them.

Low conductivity surface water overlying highly conductive groundwater results in signal to noise problems in many cases. Data must therefore be clipped to prevent artifacts; however the clipping itself can cause artifacts. Inversion must be able to accommodate the complications added by clipping.

\section{Interpretation - Case Studies}

A little local geological information usually resolves ambiguity in interpretation of the significance of electrical conductivity anomalies. Anomalies almost always correlate with groundwater salinity. Correlation with clay content is also common. Examples of use of towed geo-electric arrays are presented in Figures 6,7 and 8. Figure 6 presents a case where a submerged array has been used to suggest where water is seeping from a canal into buried river channels. Comparison with seepage under the river connected to one end of the canal is possible. Under the river, a much lower conductivity than under the canal is observed. In this environment, it suggests that seepage under the river is far more significant than seepage out of the canal. 


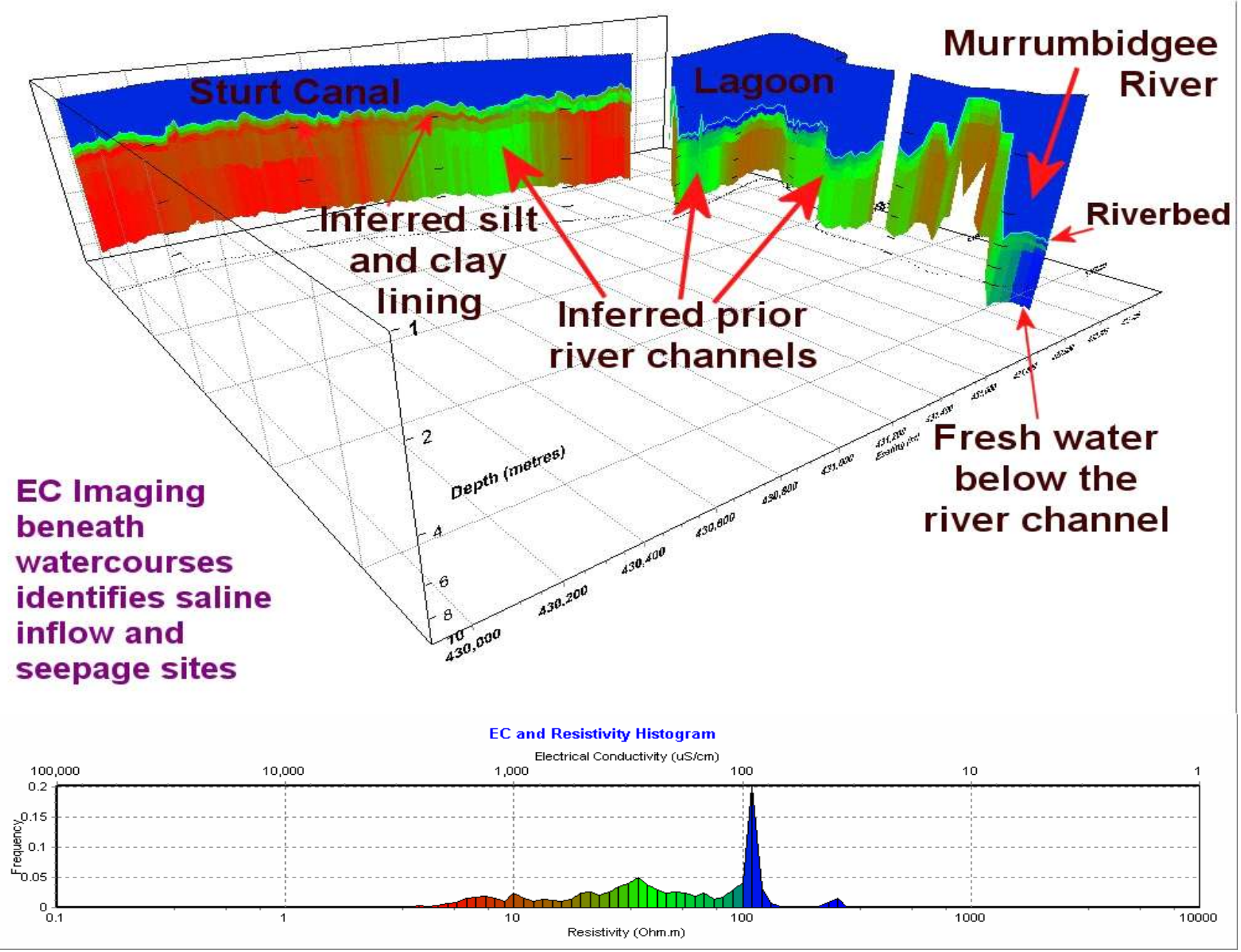

Figure 6.: Sturt Canal - Murrumbidgee Irrigaiton Area - NSW - Australia. An example of submerged geo-electric array data with intense detail right at the riverbed. Data was collected using an Iris Instruments Syscal Pro provided by Geoforce Pty Ltd. This data has been imaged simply by using an apparent resistivity formula for a submerged array in a half space along with sonar depth information, surface water resistivity and half space effective depths. The imaging procedure used is far from optimal however the level of detail produced is still impressive. Fast submerged array inversion is not yet available.

Figures 7 and 8 present a site where saline inflow into the Murray River is being prevented by salt water interception scheme (SIS) bores. Because EC reflects groundwater salinity rather than rate of saline flow, in most cases, in the vicinity of SIS bores, EC anomalies are not expected unless the SIS bores have been pumping sufficient flows to have caused a reversal of vertical groundwater flux under the river so that river water is drawn into the SIS bores. As soon as that occurs, the strata under the river become flushed with fresh river water rather than up-welling saline groundwater and a distinct EC anomaly occurs. The EC anomaly then shows the extent of strong influence of the SIS bore which in many cases is distorted by geological variations such as prior river channels. At Waikerie SIS - Murray River - South Australia, good examples of such anomalies do seem to exist. Figures 7 and 8 show such anomalies as well as another good anomaly believed to be resulting from a buried deep river channel which has left evidence of itself in the airphoto in Figure 7. Figure 8 is a close up view of the anomalies in Figure 7. 
It is believed that because the bores shown in Figures 7 and 8 are very close to the river, and because the geological strata there, that the river has incised, are permeable, distinct anomalies exist around the bores. Irrigation there has a localized impact on saline inflow into the river as is evident in Figure 7 as well as in groundwater mound anomalies.

The ribbon image in Figure 7 is a heavily smoothed 2D inversion conducted using Scott McInnes' (Zonge Engineering and Research Organization) inversion software which is very similar to Loke's Res2DInv. Figure 8 is inverted using the 1D inversion software written by the author extending the capabilities of software by Merrick (1977). The results are very similar once the 2D inversion has been horizontally smoothed to remove a resonating artifact (horizontal ripple) in the 2D inversion. The time taken to conduct the inversion however is not similar - the $1 \mathrm{D}$ inversion can be completed in minutes while one watches it being progressively imaged on a computer screen however the 2D inversion takes 10's of hours to complete. For exponentially spaced arrays, the duration of 2D inversion is greatly increased. 2D inversion of the Waikerie data was only possible because the survey was completed using a linearly spaced dipole - dipole array. Such an array has poor signal to noise ratios at greater depths and a poor distribution of effective depths as is evident in the layering in Figure 8 when compared with Figure 6 which was collected using an exponentially spaced array.

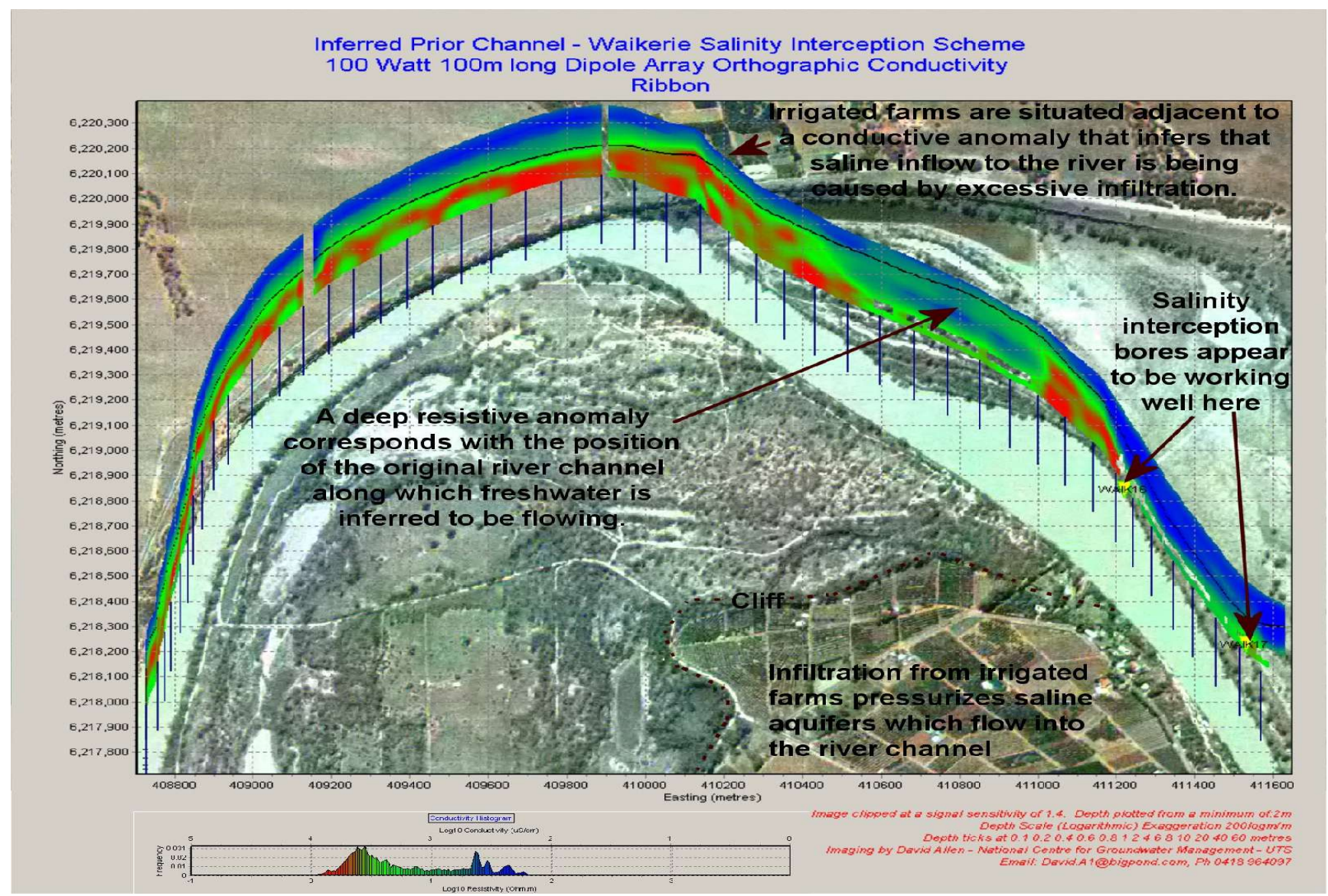

Figure 7.: Part of the Murray River within the Waikerie salt water interception scheme in which there is evidence of saline inflow related to irrigation near the river (top), a deep, buried, fresh/brackish water filled prior river channel (centre right) and freshwater flushing of strata beneath the river in the vicinity of SIS bores (far right). Note how the cliff intersects the river at the edge of the prior river channel anomaly. See Figure 8 for close up views of the anomalies. This ribbon was inverted using a 2D algorithm. 


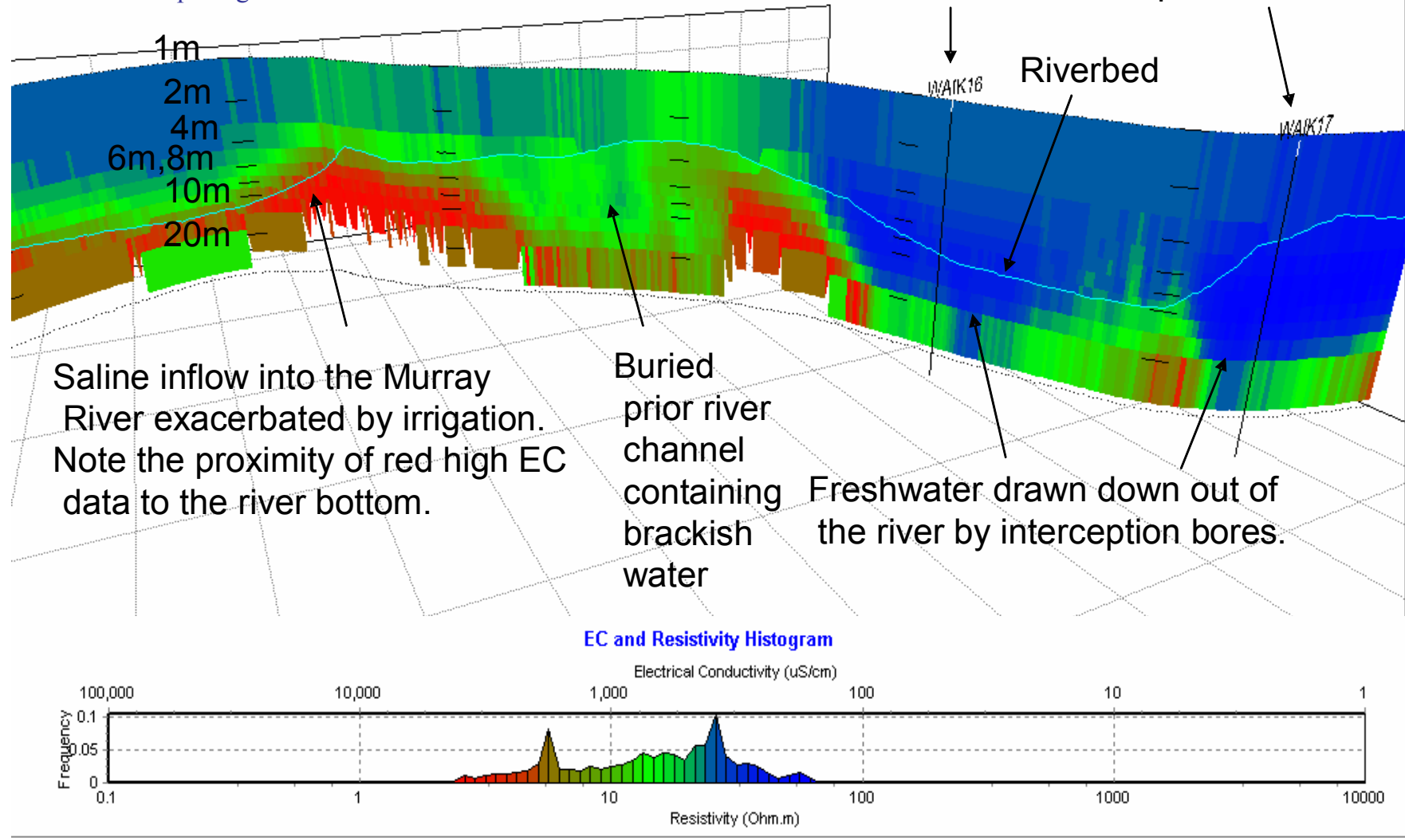

Figure 8.: Part of Waikerie SIS scheme shown in Figure 7. A close-up of anomalies resulting from a palaeochannel, and Waikerie SIS bores 16 and 17 (shown as vertical black lines). This ribbon image was generated using 1D inversion - contrast it with the 2D inversion in Figure 7. Note the thick surface layer that is a result of the poor near surface resolution of linearly spaced dipole-dipole arrays such as used at Waikerie. Note how the riverbed (aqua line) does not correspond with the base of the blue low EC anomaly near the SIS bores - the bores appear to have drawn freshwater downwards from the bottom of the river in the vicinity of the bores.

\section{Conclusion}

Towed geo-electric surveys conducted from water can be of tremendous value due to the high productivity rate attainable on water. They can focus directly on the principal sites of surface water groundwater interaction - right beneath the surface water bodies. Because electrical conductivity is very dependant on salinity, freshwater flushing of saline aquifers and saline inflow into rivers and drains can be studied in exceptional spatial detail.

The Allen Exponential Bipole Array - used either submerged or floating, is optimized for surveys on water. Compared to dipole dipole arrays traditionally used, it offers a much greater range of resolved depths including optimal resolution at the base of watercourses as well as higher signal 
strength. Compared to the Inverse Schlumberger array or Wenner array, it offers more robust, lightweight equipment design possibilities for use in water and, when used with suitable linear transmitter electrodes, better signal strength.

\section{References}

Allen, D.A., Merrick, N.P., 2003, A floating electrode array for continuous geo-electric imaging. Extended abstract in: Exploration Geophysics 2003- CD available at ASEG conference, journal published mid 2003.

Allen, D.A., Merrick N.P., 2003, Identifying fresh and saline water using hydro-geophysical imagery of strata beneath watercourses. Paper and posters presented in the proceedings of the $9^{\text {th }}$ Murray Darling Basin Groundwater Conference - Feb. - Bendigo.

Merrick, N.P., 1977, Resistivity Sounding for Groundwater. MSc of Science Thesis, University of Sydney.

\section{Acknowledgements}

The following groups are acknowledged for their contributions to the project:

- The Australian Rice Co-operative Research Centre for Sustainable Rice Production who made equipment and processing advancements possible.

- Zonge Engineering and Research Organization who provided equipment on a research rate rather than commercial rate.

- Scott McInnes - Zonge Engineering and Research Organization who completed and conveyed a similar geo-electric array appraisal for roll along imaging systems.

- Geoforce- who also provided equipment on research rates.

- Barry Porter - South Australian Department of Water, Land and Biodiversity Conservation. 